\title{
Universiteit
}

Leiden

The Netherlands

\section{Boundary layer model for vortex fingers in type II superconductors}

Saarloos, W. van; Baggio, C.; Goldstein, R.E.; Pesci, A.I.

\section{Citation}

Saarloos, W. van, Baggio, C., Goldstein, R. E., \& Pesci, A. I. (2005). Boundary layer model for vortex fingers in type II superconductors. Physical Review B, 72(6), 060503.

doi:10.1103/PhysRevB.72.060503

Version: $\quad$ Not Applicable (or Unknown)

License: $\quad$ Leiden University Non-exclusive license

Downloaded from: https://hdl.handle.net/1887/66596

Note: To cite this publication please use the final published version (if applicable). 


\title{
Boundary layer model for vortex fingers in type-II superconductors
}

\author{
Chiara Baggio, ${ }^{1}$ Raymond E. Goldstein, ${ }^{2}$ Adriana I. Pesci, ${ }^{2}$ and Wim van Saarloos ${ }^{1}$ \\ ${ }^{1}$ Instituut-Lorentz, Leiden University, PO Box 9506, 2300 RA Leiden, The Netherlands \\ ${ }^{2}$ Department of Physics, University of Arizona, 1118 E 4th Street, Tucson, Arizona 85721, USA
}

(Received 14 June 2005; published 9 August 2005)

\begin{abstract}
Propagating fingerlike patterns in type-II superconductors are studied through a boundary layer model that takes into account the coupling with the temperature of the sample. By formulating an approach based on an interfacial description for a domain of vortices, we determine the shape-preserving fronts and study the properties and scale of the patterns, such as the fingers' shape and width. We show that the formation and the characteristics of these instabilities are strictly related to the local overheating of the material and depend on the substrate temperature, in agreement with the experiments and suggestions from linear stability calculations.
\end{abstract}

DOI: 10.1103/PhysRevB.72.060503

PACS number(s): 74.25.Qt, 05.70.Ln, 89.75.Kd

The dynamics of vortices in type-II superconductors exhibits a wide variety of instabilities of thermomagnetic origin. ${ }^{1}$ Beyond phenomena such as avalanches and flux jumps, recent experiments have revealed interesting out-ofequilibrium patterns involving the formation of ramified dendritic or finger-shaped domains of vortices in $\mathrm{Nb}$ and $\mathrm{MgB}_{2}$ thin films and dropletlike patterns in $\mathrm{NbSe}_{2}$ single crystals. ${ }^{2-6}$ It is generally accepted that the nonuniform penetration of the magnetic flux is a thermomagnetic effect due to the local overheating produced by the dissipative motion of vortices. As a consequence of the increased local temperature, the pinning barrier is lowered, leading to a large-scale flux invasion and to a final nonuniform magnetic flux distribution. ${ }^{7}$

The thermomagnetic nature of the instability underlying the evolution of a flat front between the vortex and the superconducting states into narrow fingers and dendrites has been proposed in some recent theoretical models, ${ }^{8-10}$ augmented by numerical simulations and linear stability analysis. However, the shapes of the fingers, their remarkably well-defined widths between 20 and $50 \mu \mathrm{m}$, and their dependence on the substrate temperature were not obtained explicitly in this earlier work. In this paper we concentrate particularly on these finger-type growth forms and propose that they are self-organized propagating shapes with a relatively high temperature and mobility at the tip and a low temperature and mobility on the sides.

A detailed analysis for the shape of the fingers requires a more tractable mathematical model than the ones proposed previously. In particular, the formulation of an interfacial description for the vortex front is an effective and simple method to study the problem in its essential features. Local growth models have proven to be a useful tool to analyze front propagation in other physical systems, such as dendrites in crystal growth, and also magnetic flux penetration in type-I superconductors. ${ }^{11-14}$ The sharp interface limit is appropriate when the vortex density and temperature change rapidly in a layer whose thickness is thin in comparison to the radius of the curvature of the front.

In the case of a type-II superconductor, the coarse-grained density of vortices can be represented by a continuous field that decays near the interface with the vortex-free superconducting state over a distance set by the penetration depth $\lambda$.
For a type-II superconductor, $\lambda$ is of the order of $10^{2} \mathrm{~nm}$. Moreover, for the fast-moving vortex fingers of Ref. 4, the thermal decay length can become significantly smaller than the width of the domain. Therefore, there is a strong separation of scales between the domain size (typically of the order of $0.1 \mathrm{~mm}$ ) and the width of the interface. As a consequence, an interfacial description of fingerlike patterns is an appropriate and accurate approach.

We consider a thin film with the thickness $d \approx \lambda$ that is in contact with a substrate at a temperature $T_{0}$. The magnetic induction $\mathbf{B}$ is perpendicular to the plane that represents the film. By assuming a domain of vortices with a uniform density of magnetization in the bulk, we consider the approximation in which there is a constant current density $j$ only along the interface. For a more realistic description, one should account for a spatially varying current, itself derived from the long range interaction between vortices, as in Ref. 3. Since in successive experiments vortex fingers shoot into the sample at different positions, sample inhomogeneities do not appear to play an important role, so we ignore these here.

By adopting a boundary layer approximation, we represent the front between the vortices and the superconducting state by a one-dimensional curve in the plane of the film. A point on the interface is defined by its arclength coordinate $s$, a distance $r(s)$ from a fixed origin, the local Frenet-Serret frame of the tangent and normal vectors $(\mathbf{t}, \mathbf{n})$, and the angle $\theta(s)$ between the normal to the curve and the direction of propagation. The curvature of the interface is then defined by $\kappa(s)=-\partial \theta / \partial s$.

For the dynamics of the vortices we consider a local dissipative motion with a viscosity $\eta$ defined by the BardeenStephen model. ${ }^{15}$ Vortices move in the direction normal to the interface with a velocity $v_{n}(s)=\mathbf{n} \cdot \partial \mathbf{r}(s) / \partial t$ given by

$$
\eta v_{n}(s)=f\left(j, T_{i}(s)\right)\left(\frac{\phi_{0} j}{c}\right), \quad \eta=\frac{B \phi_{0}}{\rho_{f} c^{2}}
$$

where $T_{i}$ is the temperature at the interface, $\rho_{f}=\rho_{n} B / B_{c 2}$ is the flux flow resistivity and the function $f\left(j, T_{i}\right)$ gives the $E-j$ characteristic through the following dependence: 


$$
E=\rho_{f} f\left(j, T_{i}\right) j .
$$

The steepness of the electric field-current characteristic is an important feature necessary to observe these patterns. ${ }^{7}$ For the dynamics of vortices we take into account the two relevant regimes of flux flow and creep. For $j \gg j_{c}$, with $j_{c}$ the critical current density at which the $E$ - $j$ characteristic becomes linear, $E \approx \rho_{f}\left[j-j_{c}\left(T_{i}\right)\right]$. In the creep regime for $j$ $<j_{c}$ in which the vortex motion can be considered thermally activated, ${ }^{16}$ the $E-j$ relation can be approximated as $E$ $\approx \rho_{f} \exp \left\{\left[j-j_{c}\left(T_{i}\right)\right] / j_{1}\right\}$, with a flux creep rate $j_{1} \ll j_{c}$ independent of $T_{i}$ for a low- $T_{c}$ superconductor. ${ }^{17}$ The simplest approximation for the function $f\left(j, T_{i}\right)$ is thus to consider that vortices are pinned when $j_{c}$ exceeds $j$. Thus $f\left(j, T_{i}\right)$ is discontinuous with

$$
\begin{gathered}
f\left(j, T_{i}\right)=\left[1-j_{c}\left(T_{i}\right) / j\right], \text { for } j \geqslant j_{c}\left(T_{i}\right), \\
f\left(j, T_{i}\right)=0, \quad \text { for } j<j_{c}\left(T_{i}\right),
\end{gathered}
$$

where the pinning current decreases with the temperature. We linearize it as $j_{c}=j_{0}\left(1-T_{i} / T_{c}\right)$ and $j_{0}=j_{c}\left(T_{0}\right)$. In reality the current-electric field characteristic is never so sharp, and is represented by a continuous smooth function; a reasonable expression for the function $f\left(j, T_{i}\right)$, which interpolates between the two dynamical behaviors described above, is given by $^{8}$

$$
f\left(j, T_{i}\right)=\left(j_{1} / j\right) \ln \left\{1+\exp \left[\left(j-j_{c}\left(T_{i}\right)\right) / j_{1}\right]\right\} .
$$

In order to study the front dynamics, we must account for the coupling to the local temperature at the interface $T_{i}(s)$, as given by (1). As we have already mentioned, the temperature $T(\mathbf{r})$ at a point $\mathbf{r}$ of the film is enhanced by the joule heating; this is expressed by the product $\mathbf{E} \cdot \mathbf{j}$. As the system is also coupled to a substrate, we also consider the relaxation of the temperature to $T_{0}$. Therefore, the temperature field $T(\mathbf{r})$ satisfies $^{8}$

$$
C \partial_{t} T(\mathbf{r})=\nabla K \nabla T(\mathbf{r})-\left[T(\mathbf{r})-T_{0}\right] h / d+\mathbf{E}(j, T(\mathbf{r})) \cdot \mathbf{j},
$$

where $C$ and $K$ are, respectively, the heat capacitance and the thermal conductivity of the superconducting film and $h$ is the heat transfer coefficient to the substrate.

The crux of our sharp interface approximation is the idea that we can characterize the temperature field in the local system of coordinates $(\mathbf{t}, \mathbf{n})$, as $T(s, \xi)$ with $\xi$ a coordinate along the normal component, through an effective boundary layer thickness $l(s)^{14}$

$$
T(s, \xi)=T_{i}(s) \exp [-\xi / l(s)]
$$

In a co-moving frame in which the front at a point of the interface moves with a velocity $v_{n}(s), \quad \partial_{t} T_{i}=\left.\partial_{t} T_{i}\right|_{\xi}$ $-v_{n}(s) \partial_{\xi} T_{i}$. An equation for the interface temperature $T_{i}$ is then obtained by expressing the diffusion contribution in terms of the local coordinates $(s, \xi)$ and curvature $\kappa(s)$ and integrating Eq. (5) through the boundary layer

$$
\begin{gathered}
\tau_{n}(s)=f\left(j, T_{i}(s)\right) j \\
\partial_{t}\left[T_{i}(s) l(s)\right]=-\left[v_{n}(s)+\kappa(s)\right] T_{i}(s)-T_{i}(s) \\
+\alpha f\left(j, T_{i}\right) j^{2}+\partial_{s}^{2}\left[l(s) T_{i}(s)\right] .
\end{gathered}
$$

The first term on the right derives from the co-moving frame and from diffusion in the direction normal to the front, whereas the other terms represent respectively the relaxation to the substrate temperature, the heat due to dissipation, and the lateral diffusion. In this system of equations we have rescaled the variables by measuring the temperature $T_{i}$ at the interface in units of $\left(T_{i}-T_{0}\right) /\left(T_{c}-T_{0}\right)$, lengths in units of $L_{h}=\sqrt{K d / h}$, time in units of $t_{h}=C d / h$, currents in units of $j_{c}(0)$, and fields as $b=B / B_{1}, B_{1}=\left[4 \pi J_{c}(0) L_{h}\right] / c$. The only remaining parameters are the constants $\tau=4 \pi K /\left(\rho_{n} c^{2} C\right) B_{c 2} / B_{1}$ and $\alpha=\rho_{n} j_{c}^{2} d /\left[h\left(T_{c}-T_{0}\right)\right] B / B_{c 2}$.

Typical parameters of the $\mathrm{Nb}$ thin films of Ref. 2 are $d$ $\approx 0.5 \mu \mathrm{m}, \rho_{n} \approx 1.7 \times 10^{-6} \Omega$ and $C \approx 10^{-2} \mathrm{~J} / \mathrm{cm}^{3} \mathrm{~K}$. For the heat transfer coefficient $h$ and conductivity $K$ we can assume $h \approx 1 \mathrm{~W} / \mathrm{cm}^{2} \mathrm{~K}$ and $K \approx 1 \mathrm{~W} / \mathrm{cm} \mathrm{K}^{7,8}$ We thus estimate the characteristic length of our system as $L_{h} \approx 50 \mu \mathrm{m}$, and the time $t_{h} \approx 10^{-6}-10^{-7} \mathrm{~s}$.

The dimensionless constant $\alpha$ quantifies the ratio between the energy produced by joule dissipation and the heat loss to the substrate. In order to observe the instability, $\alpha \geqslant 1 .^{7}$ For a magnetic field $B \approx 20 \mathrm{mT}$ and $B_{c 2} \approx 2 \mathrm{~T}$, a critical pinning current $j_{c} \approx 10^{6} \mathrm{~A} / \mathrm{cm}^{2}, 2,4$ one finds $\alpha \approx 10-10^{2}$. The constant $\tau$ compares time scales for the magnetic field diffusion and the thermal diffusion. Using parameters estimated for a $\mathrm{Nb}$ thin film, we find $\tau \approx 10^{-1}-10^{-2}$, implying that the vortex flux density responds much faster to the inhomogeneities than the temperature. This justifies the picture of a sharpedged domain of almost constant vortex density, whose motion is coupled to a temperature that decays within a boundary layer of thickness $l(s)$.

The boundary layer thickness is derived by assuming that the curvature of the pattern is small with respect to the inner scale of the front, $\kappa(s) \ll l(s)^{-1}$. Solving the heat equation in the direction normal to the front, outside the region where the current density $j$ is present, we obtain

$$
l(s)=2\left[v_{n}(s)+\sqrt{v_{n}(s)^{2}+4}\right]^{-1} .
$$

Since we are interested in determining nontrivial fingerlike front solutions, we concentrate on the shape-preserving growth forms, such that at each point the interface moves with a constant velocity,

$$
v_{n}(s)=v_{0} \cos \theta(s),
$$

while in the frame with a fixed angle $\theta$ the fields are stationary,

$$
\left.\partial_{t}\left[T_{i}(s) l(s)\right]\right|_{\theta}=0 .
$$

The boundary layer approximation enables us to determine the shape of the fingers by reducing the problem to a single equation for the curvature of the front. Examining the relationship between the time derivative in the direction normal 
to the interface and the frame that moves with constant velocity, we obtain

$$
\partial_{t}\left(T_{i}(s) l(s)\right)=-\kappa \partial_{\theta} v_{n} \partial_{\theta}\left(T_{i}(s) l(s)\right) .
$$

Together with (7)-(9), this leads to a nonlinear differential equation for the angle $\theta(s)$. We determine the solution both for the simplified form (3) of the function $f\left(j, T_{i}\right)$ and for the expression given by (4). In both cases the problem is reduced to solving a nonlinear equation of second order for $\theta(s)$. We seek trajectories in the $\theta, \kappa, \zeta=\partial_{s} \kappa$ space that are finger solutions that start at $\theta=0$ with $\zeta=0$, and flow to the fixed point $\theta=\pi / 2, \kappa=0, \zeta=0$.

In the first case, we assume a discontinuous electric fieldcurrent characteristic. This implies also a discontinuity for the curvature $\kappa$ of the interface at a point $s^{*}$ and a value $T_{i}\left(s^{*}\right)=1-j$ in the dimensionless variable for the temperature, such that $f\left(j,\left[T_{i}\left(s^{*}\right)\right]\right)=0$. In particular, there is a sharp transition in the front dynamics at this point, since for $s$ $<s^{*}$, vortices are pinned, and the curvature vanishes with $\theta=\pi / 2$, whereas for $s \geqslant s^{*}$ the dynamical behavior is dominated by a flux flow regime. Therefore, we allow the curvature of the front to be discontinuous, but we have to impose the continuity of the physical temperature field together with its derivatives at $s^{*}$. In the region in which $v_{n}$ $=0$, the heat source $\mathbf{E} \cdot \mathbf{j}=0$, from (7) we find that the temperature field decays exponentially to $T_{0}$. By matching the boundary conditions at $s=s^{*}$ we derive two relations for curvature $\kappa$ and its derivative $\zeta=\partial_{s} \kappa$. These, together with the second-order equation for $\theta(s)$ define a unique expression for the current density $j$ as a function of the the fixed parameters $\tau$ and $v_{0}$. Finally, by shooting from the point $\theta=\pi / 2$ to $\theta$ $=0$, a unique velocity for the front is selected.

The case with a smooth function for the $E-j$ characteristic, defined by (4), does not require any restrictions on the continuity of the $T_{i}$ field. The description of the creep regime in terms of an activation barrier implies that the velocity of the vortices at the boundary for $\theta \approx \pi / 2$ vanishes exponentially as $v_{0}(\cos \theta) \approx \tau^{-1} \exp \left[\left(j-\left(1-T_{i}\right)\right) / j_{1}\right]$. As a consequence, for a finite value of the flux creep rate, the vortex velocity becomes extremely small but nonzero, so we integrate from $\theta$ $=0$ to $\theta_{0} \approx \pi / 2$. At $\theta=\theta_{0}$, we impose for the $T_{i}$ field the asymptotic value for a straight front that corresponds to the equilibrium temperature for which the heat released by the joule effect is transfered to the substrate.

Figure 1 shows the comparison of the $\theta$ profile and the temperature distribution as a function of the arclength $s$ for the cases of discontinuous and continuous current-voltage characteristic, respectively, with the same value of the tip velocity $v_{0}$ and current density $j$. As the plot shows, the curve related to the smooth current-electric field relation $f\left(j, T_{i}\right)$ overlaps in the limit $j_{1} \rightarrow 0$, with the one with a sharp function $f\left(j, T_{i}\right)$. The temperature field is larger at the tip, where vortices move faster and thus more heat is generated, whereas it vanishes as $\theta$ that approaches $\pi / 2$. In Fig. 2 we represent instead the shape of the fingers for different values of the coefficient $\alpha$ and a fixed value of the velocity $\left(v_{0}\right.$ $=1.431$ in our units $\left.L_{h} / t_{h}\right)$ that corresponds to the typical order found in the experiments $\left(v_{0} \approx 10^{4}-10^{5} \mathrm{~cm} / \mathrm{s}\right)$. The

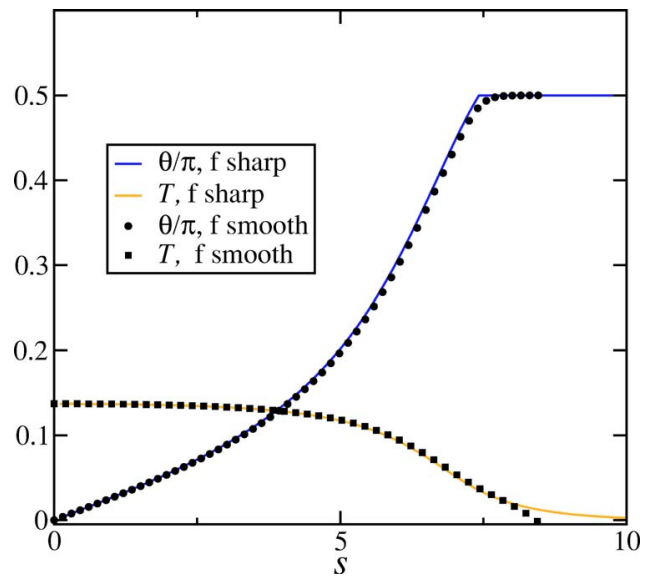

FIG. 1. (Color online) Comparison of the $\theta(s)$ and $T(s)$ fields profiles in the cases with discontinuous and continuous functions $f(j, T)$. The data correspond to the values $v_{0}=1.0193$, flux creep rate $j_{1}=0.0043, \alpha=3.9, \tau=0.1, j=0.9648$.

width of the flux filaments for a correspondent current density $j \approx 0.925 j_{c}$ varies in the range $50-150 \mu \mathrm{m}$ for $\alpha$ $=8-20$, as is shown in Fig. 3, in good agreement with the experimental studies. According to the experiments, as the substrate temperature decreases, fingers get narrower. The dependence of the width on $\alpha$ is consistent with this behavior. Indeed, $j_{c}\left(T_{0}\right)=j_{c}(0)\left(1-T_{i} / T_{c}\right)$ implies $\alpha \propto\left(T_{c}-T_{0}\right)$. Thus, the finger width decreases as $\alpha$ gets larger in agreement with our results. Taking into account the physical mechanism that triggers the instability, we can interpret this behavior in these terms: For an enhanced heat dissipation, vortices are driven faster due to the local thermomagnetic instability in the direction in which the Lorentz force is maximal, so, for the same amount of flux, the fingers are narrower. Too narrow fingers are however suppressed by thermal diffusion. This picture is consistent qualitatively also with the results of Ref. 10 .
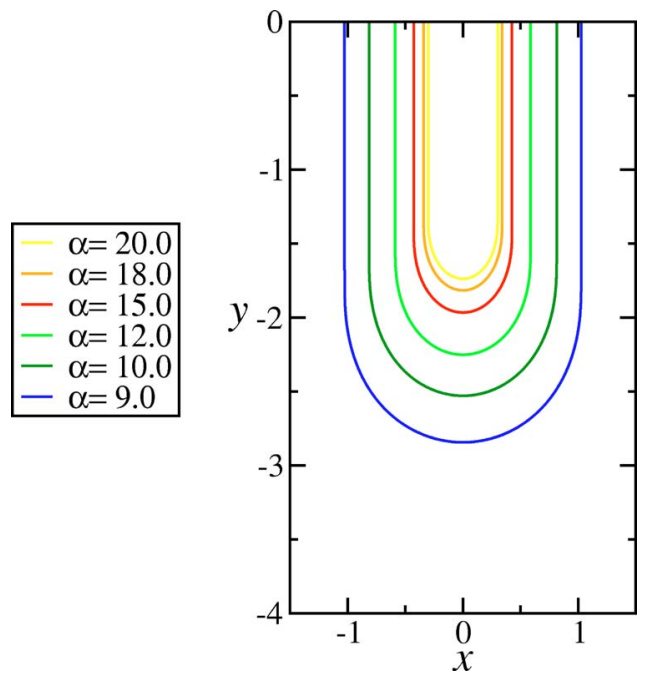

FIG. 2. (Color online) Fingers shapes in the case with smooth $f\left(j, T_{i}\right)$ for different values of the coefficient $\alpha$ for a velocity $v_{0}$ $=1.431$ in our dimensionless variables, $j=0.925, \tau=10^{-1}$ and $j_{1}$ $=0.004$. By increasing $\alpha$ the fingers become less wide. 


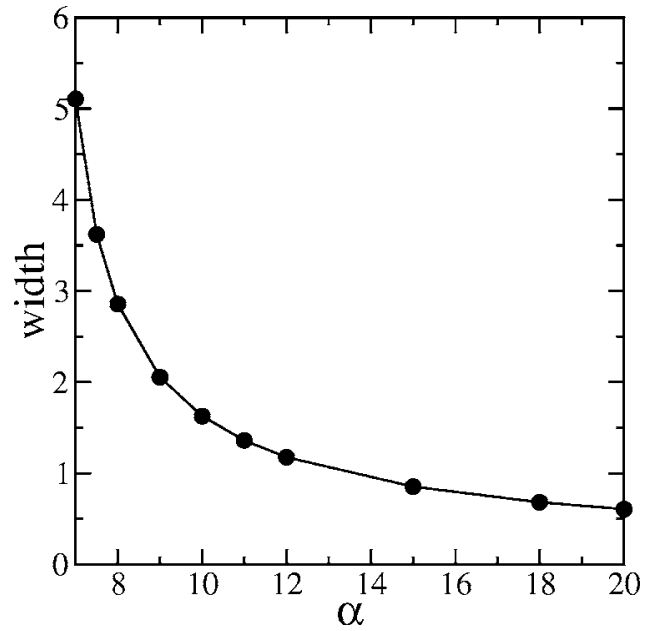

FIG. 3. Plot of the width $w$ of the fingers vs the coefficient $\alpha$ for parameter values of Fig. 2 .

Finally, we remark that while for the smooth model we find a continuous family of finger solutions, parametrized, e.g., by $v_{0}$, the discontinuous model has only solutions for a particular velocity. This discrepancy can be interpreted as a consequence of the fact that a discontinuous function $f\left(j, T_{i}\right)$ implies a "fictitious" constraint for the velocity of the $T_{i}$ field. From a more mathematical perspective, we expect that the introduction of a surface-tension-type term in Eq. (1) could lead to the "selection" of a unique shape and velocity from the family of solutions in the smooth model, in analogy with the dendrites in crystal growth or viscous fingering. However, we believe it is a delicate open issue whether such a surface-tension-type term would make sense for the vortex problem. First of all, the finger propagation is an extreme out-of-equilibrium problem. Secondly, even if it could define a positive surface tension at the interface in analogy with the case between the solid and liquid phases, ${ }^{18}$ the long-range repulsive interaction between vortices would indeed play the major role in the front dynamics. We leave this issue for the future.

We are grateful to A. T. Dorsey and P. H. Kes for illuminating discussions.
${ }^{1}$ G. Blatter, M. V. Feigel'man, V. B. Geshkenbein, A. I. Larkin, and V. M. Vinokur, Rev. Mod. Phys. 66, 1125 (1994).

${ }^{2}$ C. A. Duran, P. L. Gammel, R. E. Miller, and D. J. Bishop, Phys. Rev. B 52, 75 (1995).

${ }^{3}$ T. H. Johansen, M. Baziljevich, D. V. Shantsev, P. E. Goa, Y. M. Galperin, W. N. Kang, H. J. Kim, E. M. Choi, M.-S. Kim, and S. I. Lee, Europhys. Lett. 59, 599 (2002).

${ }^{4}$ M. S. Welling, R. J. Westerwaal, W. Lohstroh, and R. J. Wijngaarden, Physica C 411, 11 (2004).

${ }^{5}$ M. Menghini, R. J. Wijngaarden, A. V. Silhanek, S. Raedts, and V. V. Moshchalkov, Phys. Rev. B 71, 104506 (2005).

${ }^{6}$ M. Marchevsky, L. A. Gurevich, P. H. Kes, and J. Aarts, Phys. Rev. Lett. 75, 2400 (1995)

${ }^{7}$ G. R. Mints and A. L. Rachmanov, Rev. Mod. Phys. 53, 551 (1981)

${ }^{8}$ I. Aranson, A. Gurevich, and V. Vinokur, Phys. Rev. Lett. 87, 067003 (2001).

${ }^{9}$ I. S. Aranson, A. Gurevich, M. S. Welling, R. J. Wijngaarden, V.
K. Vlasko-Vlasov, V. Vinokur, and U. Welp, Phys. Rev. Lett. 94, 037002 (2005).

${ }^{10}$ A. L. Rakhmanov, D. V. Shantsev, Y. M. Galperin, and T. H. Johansen, Phys. Rev. B 70, 224502 (2004).

${ }^{11}$ A. T. Dorsey, and R. E. Goldstein, Phys. Rev. B 57, 3058 (1998).

${ }^{12}$ H. Frahm, S. Ullah, and A. T. Dorsey, Phys. Rev. Lett. 66, 3067 (1991).

${ }^{13}$ F. Liu, M. Mondello, and N. Goldenfeld, Phys. Rev. Lett. 66, 3071 (1991).

${ }^{14}$ E. Ben-Jacob, N. Goldenfeld, J. S. Langer, and G. Schön, Phys. Rev. A 29, 330 (1984).

${ }^{15}$ J. Bardeen and M. J. Stephen, Phys. Rev. 140, A1197 (1965).

${ }^{16}$ P. W. Anderson, Phys. Rev. Lett. 9, 309 (1962).

${ }^{17}$ A. M. Campbell and J. E. Evette, Adv. Phys. 21, 119 (1972).

${ }^{18}$ A. Soibel, E. Zeldov, M. Rappaport, Y. Myasoedov, T. Tamegai, S. Ooi, M. Konczykowsky, and V. B. Geshkenbein, Nature (London) 406, 282 (2000). 